\title{
De la victoria de un candidato independiente a los desafíos en la eficacia del desempeño gubernamental: el caso del estado de Nuevo León, México*
}

\author{
From the Victory of an Independent Candidate to the Challenges in the Government's Performance Efficacy: The \\ Case of the State of Nuevo León, Mexico
}

Laura Nelly Medellin Mendoza ${ }^{\text {a }}$

Universidad Autónoma de Nuevo León, México

lauramedellin76@hotmail.com

ORCID: http://orcid.org/0000-0003-1049-1635
DOI: https://doi.org/10.11144/Javeriana.papo23-2.vcid Redalyc: http://www.redalyc.org/articulo.oa?id=77757839004

Fecha de recepción: 17 Marzo 2018

Fecha de aprobación: 25 Junio 2018 Fecha de publicación: 18 Diciembre 2018

\section{Resumen:}

Se analiza la victoria de un candidato independiente que ganó un gobierno subnacional excluyendo al sistema tradicional de partidos políticos. Como fue una experiencia inédita en México, se toman en cuenta las condiciones del sistema político, caracterizado por la dominación de un partido hegemónico cuya capacidad para minimizar la representación política fue la constante, hasta que gradualmente el proceso de liberalización política por la vía electoral pudo permitir el pluralismo político. En la reforma nacional electoral del año 2014 se contó con la posibilidad de tener candidaturas independientes, y el estado de Nuevo León también incluyó la figura. Por tanto, en el análisis del primer gobierno independiente del estado de Nuevo León se considera la variable antecedente de cómo fue la ruta electoral ganadora que a través, fundamentalmente, de una plataforma de redes sociales y no por la vía convencional de hacer campañas electorales, se logró una victoria sin precedentes en la historia local y nacional. Posteriormente, centrándose solo en dos variables para medir eficacia política gubernamental, se muestra evidencia empírica para analizar los resultados en materia de corrupción y seguridad pública. Finalmente, se incluye el contexto de la salida de la gubernatura y las conclusiones en el primer balance de gobierno.

Palabras clave: sistema político, legislación electoral, candidaturas independientes, gobierno subnacional.

\section{Abstract:}

The victory of an independent candidate who won the subnational election and excluded the traditional system of political parties is analyzed herein. As an unprecedented case in Mexico, it is analyzed considering the conditions of the political system characterized by a hegemonic political party dominating everything, where their capability to minimize the political representation has been a constant trait. Notwithstanding, the political liberalization process gradually managed to allow the political pluralism through the elections. The national election reform in 2014 provided the possibility to have independent candidates and the State of Nuevo León implemented it. Therefore, the analysis of the first independent government in Nuevo León examines the variable precedent to the wining electoral route. Thanks to a campaign basically through a social networking platform instead of a conventional election campaign, the candidate achieved an unprecedented victory both in the local and national history. Next, focused only on two variables measuring the governmental political efficacy, some empirical evidence is shown in order to analyze the results regarding the public security and corruption issues. Finally, this work addresses the context of the outgoing governor and provides the conclusions from the first governmental balance.

Keywords: political system, election laws, independent candidacy, subnational government.

\section{Introducción}

El presente trabajo se propone recapitular la experiencia del primer estado de la República mexicana que es gobernado por un candidato independiente, Jaime Rodríguez Calderón, El Bronco, quien resultó triunfador en las elecciones de junio del 2015 para la gubernatura del estado de Nuevo León. Esto fue

Notas de autor:

a Autor de correspondencia: lauramedellin76@hotmail.com 
posible por la modificación a la legislación electoral nacional y desde luego local que permite la inclusión de la candidatura independiente en la competencia electoral. Para ello, la crítica antecedente al sistema de representación política dominado por los partidos políticos fue fundamental para posicionar la discusión sobre las candidaturas independientes, pero también el escenario fue abonado por el desempeño de los gobiernos antecesores.

En ese sentido, en nuestro primer apartado señalamos brevemente las bases de interpretación del sistema político mexicano y subnacional, en el que a partir de un modelo de partido hegemónico se generó una representación política limitada. Sin embargo, el modelo de liberalización política por la vía electoralista permitió gradualmente el avance del pluralismo en la representación política, hasta llegar en el 2014 con la legalidad de las candidaturas independientes en el sistema electoral.

En un segundo apartado, hacemos un énfasis en las condiciones inéditas que marcaron la ruta electoral triunfadora del candidato independiente a la gubernatura en el 2015, específicamente en el marketing electoral utilizado en redes sociales que desbancó a los otros candidatos del sistema de partidos con dominancia del Partido Revolucionario Institucional (PRI) y el Partido Acción Nacional (PAN). Sin embargo, cabe aclarar que este tipo de marketing aún no se encuentra regulado por la autoridad electoral. En un tercer apartado, mencionamos las condiciones que han marcado, a casi tres años de gobierno, un balance de los resultados de la eficacia política gubernamental del llamado gobierno independiente, principalmente en dos variables determinadas por los desafíos precedentes en la gestión gubernamental - y como un reclamo recurrente en la campaña electoral del 2015-, como fueron la lucha por la corrupción y el estado de la inseguridad pública. Advertimos que estas dos variables no absolutizan ni resumen el análisis general del gobierno, que puede tener otro tipo de resultados, incluso hasta positivos según la perspectiva que se adopte. No obstante, nuestro interés no es hacer un balance general — dado que constitucionalmente aún no termina su mandato - sino centrarnos en aquellas que han representado un desafío político para la eficacia gubernamental desde la perspectiva de la demanda ciudadana mostrada en campaña y que son verificadas también a partir de la Encuesta Nacional de Victimización y Percepción sobre Seguridad Pública (Envipe) 2016 y 2017.

\section{El sistema político subnacional y la introducción de las candidaturas independientes}

México es uno de los países latinoamericanos que tuvieron un arreglo de sistema político autoritario tras la hegemonía ideológica del nacionalismo revolucionario anclado estructuralmente en el denominado partido oficial (PNR, PRM, PRI) desde 1929. En este arreglo, el partido oficial tuvo como jefe máximo al presidente de la República. Esta figura tuvo amplias atribuciones constitucionales y también fue el árbitro en las mediaciones políticas y sociales del corporativismo. Aunque existía la oposición partidista, esta no llegó a ser representativa. La cooptación, la represión y el fraude electoral fueron los tres elementos funcionales para conservar el sistema político autoritario (Reveles, 2008, p. 173).

Como señala Reveles (2008), México se caracterizó por tener un sistema de partido hegemónico desde 1929, debido a: "los triunfos electorales del partido hegemónico, amplios y permanentes b) la debilidad de la oposición, producto en buena medida de su integración a la institucionalidad vigente, y c) la falta de condiciones de competencia real" (p. 174).

De acuerdo con los especialistas de la transición mexicana (Becerra, Salazar y Woldenberg, 1996; Pérez, Puga y Díaz-Santana, 2009; Woldenberg, 2012), en su ruta de liberalización política hubo diversas reformas electorales que permitieron gradualmente desde 1990 tener mayor capacidad de inclusión de partidos distintos al oficialista, con victorias para la oposición en los estados desde la última década del siglo XX (Espinoza, 2000; Hernández, 2003, 2008). En el año 2000, llegó por primera vez la alternancia en la 
presidencia de la República, y particularmente en el estado de Nuevo León en el año de 1997, con el PAN, de estirpe conservadora.

Como se sabe, el PAN se caracterizó en sus años de oposición por su lucha contra el fraude electoral y abrir brecha en el reemplazo interpartidario, primero en el ámbito municipal, y generar un empuje electoral que posibilitó que el empresario Fernando Canales Clariond, quien gobernó de 1997 al 2003, fuera gobernador del estado de Nuevo León (Medellín, 2011).

Después del interludio panista, se efectuó la segunda alternancia, en la que el PRI regresó a gobernar el estado por dos sexenios: 2003-2009 y 2009-2015. Sin embargo, la capacidad de gobierno estuvo marcada en el primero - presidido por Natividad González Parás - por una marcada inseguridad pública y en el segundo - en cabeza de Rodrigo Medina de la Cruz - por el endeudamiento y la corrupción, lo que comprometió no solamente la figura del gobernador, sino a su familia y funcionarios del gabinete. Estos dos últimos periodos de gobierno produjeron un hartazgo hacia el sistema de partidos locales, sobre todo porque ante la marcada crisis de inseguridad, su capacidad de responsiviness fue baja, como se ha mostrado en otro estudio (Medellín, 2017).

Sin embargo, como hemos mencionado, desde la década de 1990 hubo reformas electorales que democratizaron tanto la ciudadanización del órgano electoral como la profesionalización de todas las etapas del proceso electoral. Pero fue solo en mayo del 2014 que se promulgó la legislación secundaria, en la cual se incluyó un apartado específico para regular el funcionamiento de las candidaturas independientes (Campos, 2014, pp. 80-92) ${ }^{\mathrm{i}}$. Esta inclusión viene de la propuesta de otorgar derechos políticos a los ciudadanos por fuera de los canales establecidos por los partidos políticos ya que, como señala De la Peza (2007), "la crítica hacia los sistemas que hacen descansar en los partidos políticos los procesos de conformación de la voluntad estatal, y su monopolio en la postulación de candidatos a los cargos electivos es cada día más uniforme y constante $[\ldots]$... (p. 614).

En el ámbito académico se han hecho diversos estudios que dan cuenta del análisis jurídico desde el punto de vista de su constitucionalidad (A. Ramos, 2016), o bien análisis que atienden las otras experiencias en los casos regionales (Del Toro, 2001; González, 2015; Soto, 2014). En el caso de Nuevo León, aunque el tema fue retomado por las organizaciones civiles locales que pedían su inclusión desde tiempo atrás, fue más bien la homologación de la ley federal lo que motivó principalmente que se incluyera en la legislación electoral también en el 2014 (Comisión Estatal Electoral, 2015c).

Esta reforma a la legislación electoral permitió a un outsider del PRI, Jaime Rodríguez Calderón, El Bronco, la posibilidad de inscribirse en el proceso electoral como candidato independiente. Rodríguez Calderón fue militante priista por 33 años. Entre otros cargos públicos, como ser diputado local, fue alcalde del municipio conurbado de García (2009-2012), donde sobrevivió a dos intentos de homicidio por parte del grupo criminal de Los Zetas, que tuvieron en esta etapa el control del narcomenudeo en la zona.

Previamente a la toma de su protesta como alcalde de García, su hija menor fue secuestrada y su hijo mayor fue encontrado muerto, presuntamente tras un intento de secuestro (Campos, 2015a, p. 68). También tuvo que enfrentar, como alcalde, el asesinato de su primer secretario de Seguridad Publica, quien se atrevió a limpiar la corporación de polizetas ii (Campos, 2015a, pp. 70-72). A partir de esta coyuntura, estableció desde su administración un contacto directo con sus gobernados para la denuncia de la venta de droga en el municipio.

Cuando se decidió a ir por la gubernatura de Nuevo León, renunció al partido en el 2014, al no encontrar condiciones de real competencia intrapartidista, al igual que otros destacados militantes. Y en su carta de renuncia afirmó:

He decidido unirme a millones de mexicanos que han llegado al límite de la paciencia. Y que hoy, encabronados, muy molestos exigen cambiar los viejos modos políticos del partido y del propio sistema. Coincido con ellos, porque pienso y siento lo mismo que ellos [...] Nunca quise ser candidato del PRI, lo dije siempre. Hoy quiero ser un candidato a dirigir un movimiento que permita que los ciudadanos libres, independientes podamos congregarnos. (Citado en Campos, 2015a, pp. 98-99) 
A través de la plataforma Cerebro, Corazón y Carácter, Rodríguez Calderón estructuró su candidatura a la gubernatura del estado. Uno de los requisitos más cuestionados en la legislación electoral era que para lograr el registro de una candidatura independiente se necesitaba el respaldo de al menos 3\% de una lista nominal de 3.443.131 ciudadanos registrados en el estado, es decir, de 103.249 votos en un plazo de 49 días (Mendoza, 2014). La estructura de apoyo de El Bronco, capitalizada a través de las redes sociales, logró reunir 394.182 firmas $^{\mathrm{iii}}$. Finalmente, en marzo del 2015 se hizo legal su registro como candidato independiente a la gubernatura.

\section{La campaña: la inequidad en la legislación electoral y la ganancia en las redes sociales}

Aunque en términos nacionales fue en el proceso electoral del 2012 en el que se instaló por primera vez, aunque de forma incipiente, el uso de las redes sociales para las campañas electorales a la Presidencia de la República, como lo dan cuenta diversos estudios (Castillo, 2014; R. Moreno, 2016), en el año electoral del 2015 fue ineludible contar con una estructura virtual más sólida dentro del war room de los partidos políticos en competencia. Este era un escenario completamente nuevo a la forma tradicional de comunicación política en las campañas electorales. Como se ha señalado, las campañas electorales: "[...] [son] el momento que mejor concentra la formación de la voluntad de los electores" (Becerra, 1997, p. 8), ya que de acuerdo con otra visión compartida, "[...] eslabonan, condensan y expresan múltiples aspectos de las relaciones sociopolíticas [...] sujetas a posibilidades y límites que derivan de la organización y estructura de cada partido político" (Tejera, 2003, p. 11).

Ahora bien, la manera convencional de formalizar una campaña electoral ha sido sobre todo a través de propaganda política cara a cara, pegatinas, además de spots en radio y televisión. Históricamente, han existido campañas negativas y de contraste contra el principal opositor o favorito en las encuestas que la ley electoral ha tratado de regular — reformas del 2007 y del 2014-, además de la poderosa industria financiera que se erige en los medios de comunicación, principalmente en televisión, en tiempos electorales.

El estado de Nuevo León no fue ajeno a esta forma de organizar campañas de manera habitual, pero como hemos señalado, justamente el año del 2015 marcó el viraje tradicional de la comunicación política-electoral.

Cabe señalar que al inicio del proceso de elección a la gubernatura, el análisis político local no preveía que un candidato independiente tendría algún tipo de ventaja en un escenario controlado por un marcado bipartidismo PRI-PAN, asentado históricamente desde las elecciones de 1988 tanto en la gubernatura como gracias a las victorias para el congreso local, como se ha mostrado anteriormente (Medellín y Murillo, 2015, pp. 17-21) iv.

El control político en la entidad ha sido desde la etapa de partido hegemónico de mayoría legislativa para el PRI o en la etapa de la alternancia para el PAN. Y en este sentido, las campañas electorales de estos dos partidos mayoritarios para el año de 2015 se estructuran de manera convencional, apoyadas como veremos más adelante en los medios de comunicación locales, particularmente televisión.

Según una encuesta realizada por Heras Demotecnia, en el mes de febrero la candidata de Alianza por tu Seguridad - conformada por PRI, PVEM, Nueva Alianza y Partido Demócrata - Ivonne Álvarez tenía una preferencia de 39\% en la intención de voto. Por su parte, Felipe de Jesús Cantú, candidato del PAN contaba con el $28 \%$ de las preferencias. El resto de los aspirantes en conjunto sumaban el $33 \%$. Cabe resaltar que en esta encuesta no figuró Jaime Rodríguez, a pesar de su anuncio dos meses antes de su intención de ser candidato independiente (Mendoza, 2014). Álvarez, la candidata oficialista, fue privilegiada principalmente por las televisoras locales, radio y prensa al destinarle mayor cobertura que el resto de sus contrincantes (Campos, 2015a, p. 138; M. Ramos, 2015). 
Como se ha señalado en un estudio previo, pese a este tratamiento de las televisoras con la candidata del PRI, esto no detuvo

[...] el incremento en la popularidad del candidato independiente Jaime Rodríguez Calderón "El Bronco" aunque no contó oficialmente con las mismas prerrogativas en términos de financiamiento [esto cambiaría más adelante] y de acceso a los medios de comunicación [esto permaneció igual] como los partidos políticos. (Berumen y Medellín, 2016, pp. 67-68)

$\mathrm{Al}$ ser un outsider del PRI, rápidamente se situó a partir de una estrategia antisistema, un "político incorrecto", como el mismo se definió en una entrevista para la prensa española (Martínez, 2015).

La legislación electoral del 2014 contempla un tratamiento dispar para los candidatos independientes frente al aceitado sistema de financiamiento a los partidos políticos. De acuerdo con el artículo 217 fracción II, el acceso a los tiempos de radio y televisión a los candidatos independientes registrados se contempla de "forma conjunta", esto es, como si se tratara de un partido político de nuevo registro. El mismo tratamiento está en lo relativo al financiamiento público dispuesto en el artículo 217 fracción III, ya que el monto es distribuido entre el total de número de candidatos registrados. Por otra parte, la legislación marca que los candidatos independientes tienen la posibilidad de acuerdo con el citado artículo 217 fracción IV, de obtener financiamiento privado, siempre y cuando no rebasen el monto permitido a los partidos políticos.

En otras palabras, los tiempos de acceso a radio y televisión además del dinero para la campaña son una "bolsa común" que comparten todos los registrados como independientes. De acuerdo con lo informado por la Comisión Estatal Electoral, en esta elección se presentaron solamente veintidós candidaturas independientes repartidas en todos los cargos de elección popular. En términos reales, la cantidad, que ascendió a 1.151 .139 pesos, se dividió en tres bolsas iguales por tipo de cargo de elección popular. Particularmente, al candidato independiente Jaime Rodríguez Calderón solo le correspondieron 383.329,46 pesos para hacer campaña (Comisión Estatal Electoral, 2015b, p. 34), es decir, menos del 1\% de los topes de campaña referidos a los partidos políticos (Estrada, 2015). Ante esa situación, Rodríguez Calderón recurrió sobre todo a financiamiento privado, que le fue reconocido como legal por el órgano electoral a casi unos días de la fecha de elección (7 de junio), con un tope de hasta 49.929.949,27 (Comisión Estatal Electoral, 2015b, p. 34).

Finalmente, el candidato independiente erogó en campaña, de acuerdo con un reporte técnico de fiscalización del Instituto Nacional Electoral, el monto de 8.907.441,01 pesos. Esta cifra fue validada después en la resolución del Tribunal Electoral del Poder Judicial de la Federación, que confirmó el triunfo definitivo de Rodríguez Calderón como gobernador del estado de Nuevo León (Tribunal Electoral del Poder Judicial de la Federación, 2015, pp. 104-105). La votación electoral definitiva fue: Jaime Rodríguez Calderón 1.020.552 votos (48,82\%), Ivonne Álvarez 498.644 (23,85\%) y Felipe de Jesús Cantú 466.543 votos (22,32\%) (Comisión Estatal Electoral, 2015a).

La ventaja competitiva de Rodríguez Calderón residió en una estrategia de campaña desplegada en redes sociales, en particular en Twitter, Facebook y Youtube, y mínimamente, por no decir casi nula, en la estrategia convencional de campañas electorales, ya que como hemos remarcado antes, las prerrogativas del financiamiento eran reducidas.

Un estudio previo en la campaña del candidato ganador señala a este respecto:

[...] el análisis de las redes sociales ha mostrado que en el caso de Nuevo León el marketing político-digital jugó un papel importante por lo menos en la campaña de Rodríguez Calderón que basó su estrategia en personalizar el mensaje haciendo que las barreras espacio-tiempo desaparezcan por completo. (Berumen y Medellín, 2016, p. 83) ${ }^{\mathrm{v}}$

En la parte medular del estudio se afirma que el uso intensivo, por parte de los usuarios de las redes sociales, de la propaganda vertida por este medio del candidato independiente, fue muy superior que lo logrado por el resto de sus contrincantes: 
Los resultados en comparación son interesantes. En resumen, el candidato de Acción Nacional quedó en tercer lugar tanto en votación electoral, como en sus niveles de interacción en redes sociales frente a los resultados de los otros contendientes. Tiene un promedio total de 193.773 interacciones frente a su votación electoral definitiva que fue de 466.543. Es decir, tuvo más votos que interacciones, prevaleció el voto duro panista más que votantes interesados en su plataforma. Los resultados son contrastantes con Ivonne Álvarez, ya que ella sumó un promedio total de 1.197 .986 interacciones, frente a su votación electoral definitiva de 498.644. Contrario al candidato de Acción Nacional, su perfil tuvo más interacciones pero menos votos [...] Por último, en el caso de Jaime Rodríguez, mientras que su votación definitiva fue de 1.020.552, sus niveles de interacción en las redes fueron bastante superiores, alcanzando una cifra de 5.114.083. Podemos asumir que la campaña del Bronco fue un fenómeno notorio de las redes sociales para una candidatura que no montó su campaña en medios convencionales y aun así lograr una cifra superior de votación frente a candidatos que sí tenían una amplia campaña en medios convencionales y una estructura partidista que movilizara el voto duro antes y durante el día de la votación. (Berumen y Medellín, 2016, p. 81)

Otras de las líneas sugeridas también señalaron que la victoria del candidato independiente fue promovida necesariamente por grupos de empresarios poderosos que financiaron su campaña, cuando se rompió un acuerdo histórico con el PRI (Nuncio, 2015); otras voces incluso más atrevidas, provenientes de fuentes periodísticas, señalan la posibilidad de que el narcotráfico esté relacionado con el financiamiento, luego de que fuera detenido en Estados Unidos el fiscal del estado de Nayarit, Edgar Veytia, acusado de elaborar y traficar droga, además de proteger al Cártel de Jalisco Nuevo Generación. Rodríguez Calderón como candidato anunciaba que recibía ayuda de amistades en Nayarit; ya como gobernador, no desmintió su amistad con Veytia (Redacción El Universal, 2017).

A continuación revisamos el trayecto del desempeño gubernamental a casi tres años de su inicio; advertimos la ausencia en este tiempo de una estrategia clara y definida para generar una eficacia política y generar una percepción de confianza sostenida favorablemente en el electorado que votó por él.

\section{De las promesas de campaña a la dura realidad: las demandas de lucha contra la corrupción y la inseguridad pública}

En teoría de ciencia política, la capacidad de respuesta de las autoridades políticas a los problemas públicos de manera oportuna y conveniente es lo que valida a los gobiernos eficaces. Así, dicho en otros términos, la eficacia política gubernamental es "[...] la posibilidad real para alcanzar los objetivos contraídos con la sociedad en los tiempos deseables y con ello mantener la confianza política, la credibilidad, la aceptación ciudadana en las autoridades, que no es otra cosa que la legitimidad democrática” (Alcántara, 1995, p. 15).

Por eficacia política gubernamental nos referimos a los resultados del gobierno, de sus programas, políticas públicas y estrategias que tienen la intención de beneficiar a la sociedad, a la población abierta. Se esperaría que los gobiernos independientes, al provenir de una candidatura que no parte de la misma confección genealógica de los partidos políticos inscritos en la lógica del oligarquismo, tan bien descrito por Michels (1969), tendrían una mayor cercanía con los problemas concretos de la ciudadanía y celeridad por cumplir las metas, ya que no estarían ligados a intereses oligárquicos y de corrupción ${ }^{\text {vi }}$. Gramsci señala que los partidos políticos son la fuerza unificadora de la clase en el plano político-cultural, ya que en las tareas del partido tienen un alcance de "[...] formación de una voluntad colectiva nacional-popular de la que el moderno Príncipe [el partido] es precisamente la expresión activa y operante, y [sic] reforma intelectual y moral” (Gramsci, 1999, p. 173). En ese sentido, la presencia de candidatos independientes que aspiren al poder representa una ruptura con los moldes tradicionales de agregación de intereses que representan los partidos políticos, que específicamente son portadores de un tipo de voluntad ideológico-organizativa. En ese sentido, siguiendo a Gramsci, los candidatos independientes que llegan a ser gobierno tendrían teóricamente que ofrecer una nueva hegemonía, entendida como: 
[...] construcción que permita el paso a una esfera de dirección intelectual y moral [...] la clase hegemónica debe ser una clase principal de la estructura de la sociedad, que pueda aparecer como la clase progresiva que realiza los intereses de toda la sociedad. (Campione, 2014, p. 93)

Así, la nueva hegemonía de un gobierno independiente tendría que dar resultados también eficaces en política pública. Sin embargo, en la medición de los resultados de la eficacia nos concentraremos en las que tuvieron un desafío político planteado por la misma exigencia en las campañas electorales por parte de la sociedad nuevoleonesa, como son (a) corrupción y (b) inseguridad pública. Esto también se define en las encuestas Envipe-Inegi tanto del 2016 como del 2017, en las cuales los ciudadanos de dieciocho años o más consideraron que las principales preocupaciones eran, en primer lugar, inseguridad $(71,4 \%)$, y en segundo lugar corrupción (38,5\%), en el 2016, e inseguridad (72\%) y corrupción (36\%), primer y segundo lugar en el 2017 (Envipe, 2016, 2017).

El desafío político está definido entonces, de acuerdo con las categorías de Morlino (1985, p. 220), por: (a) la intensidad con que se plantea la demanda —intensidad de los temas durante la campaña electoral—; (b) la importancia que tienen los grupos sociales políticamente relevantes promotores del desafío -ciudadanía, organizaciones civiles-; y (c) la sustancia de la exigencia que puede ser más o menos fácil de atender con respecto a los problemas que comporta - procesamiento a funcionarios públicos acusados de corrupción, reducción de los números crecientes de inseguridad pública-.

En lo que respecta al primer inciso, se puede señalar en términos claros que a pesar de la necesidad de manifestar un discurso enérgico en la campaña electoral, de que iba a meter a la clase política corrupta a la cárcel, este proceso ha sido más bien largo y con resultados concretos casi nulos.

"Se les acabó la fiesta a los bandidos", esta fue la famosa frase que pronunció Rodríguez Calderón en ocasión de su toma de protesta como gobernador de Nuevo León en octubre del 2015 (Campos, 2015b). En el mismo acto, el gobernador saliente, Rodrigo Medina de la Cruz, literalmente salió por la puerta de atrás, abandonando el acto protocolario lo más pronto posible, incluso olvidando entregarle las llaves del Palacio de Gobierno al nuevo gobernador, evitando por supuesto todo posible cuestionamiento a su gestión. La clara referencia era obviamente a su figura y su administración que había sido cuestionada muy duramente por corrupción y enriquecimiento ilícito de su familia. Este fue el tema principal durante la campaña, no solamente referido por Rodríguez Calderón, sino prácticamente por todos los candidatos a la gubernatura, incluida la priista Ivonne Álvarez cuando no lo quedó más remedio que desmarcarse de la "ola de la corrupción medinista”. Esto es importante destacarlo porque una de las críticas más fuertes que ha tenido el ahora gobernador porque justamente "meter a la cárcel a Medina" fue la mayor promesa de campaña del Bronco.

Aunque el gobierno de Rodríguez Calderón tuvo un bono electoral incuestionable, ha sido su falta de eficacia política para atender los problemas de la sociedad nuevoleonesa, el marcaje principal de este periodo de evaluación gubernamental. Así, desde el inicio, la integración de su equipo de gobierno fue cuestionada por tener personajes fuertemente ligados tanto al PAN como al PRI, es decir, no fueron ciudadanos "químicamente neutros" como había prometido en campaña.

En términos de la llamada eficacia política, no solo no ha podido meter a la cárcel al exgobernador, dado que ha sido un proceso jurídico que se pierde entre recursos legales y amparos por parte de la defensa del exgobernador Medina. Cuando estuvo a punto de hacerlo efectivo, el interfecto solo pisó por unas horas la cárcel, gracias a un amparo federal concedido (Lastiri y Carrizales, 2017) ${ }^{\text {vii }}$.

Como hemos señalado anteriormente, la Operación Tornado se aproximaba sin demasiados problemas a su objetivo esencial, Rodrigo Medina, desde que a principios de junio del 2016 la Subprocuraduría Anticorrupción anunció que procedería penalmente contra el exgobernador y sus allegados. La investigación por presuntos desvíos por 3600 millones de pesos en incentivos a la armadora Kia involucra a treinta excolaboradores, amigos y familiares de Medina. De hecho, Rodolfo Gómez Acosta, extesorero del estado, quedó vinculado a proceso por tres delitos derivados del caso Kia: daños contra el patrimonio del Estado y 
municipios, peculado y ejercicio indebido de funciones públicas, con una posible pena máxima de 36 años y sin derecho a fianza (Medellín, 2016a).

La ineficacia para la debida integración de los expedientes judiciales tiene como consecuencia directa que el exgobernador Medina haya permanecido solo once horas en la cárcel y Gómez Acosta siga libre gracias a amparos concedidos.

El caso fue visto como una vergüenza nacional, porque aquí la única eficacia jurídica mostrada fue por parte de la defensa del exgobernador y sus principales operadores, aunque esto en cualquier momento puede tener un giro y efectivamente llevar al exgobernador a la cárcel. Comparados con otros sistemas de justicia estatales que sí han librado efectivas órdenes de aprehensión contra sus exgobernadores, el caso de Medina en Nuevo León sigue estando en términos de la eficacia gubernamental "congelado".

En general, podemos señalar que en el primer corte de caja en estos primeros tres años de gestión han existido por lo menos veintidós renuncias de funcionarios de primer y segundo nivel. Las bajas más polémicas son las de Fernando Elizondo Barragán en la Coordinación Ejecutiva de la Administración Pública de Nuevo León. También los titulares de la Secretaría del Trabajo, en Educación y en la Procuraduría de Justicia del Estado de Nuevo León (Carrizales, 2017).

El nombramiento de Fernando Elizondo Barragán no estuvo exento de dificultades desde el inicio y fue visto como una condición de los grupos empresariales que apoyaron al entonces candidato Rodríguez Calderón. Su puesto fue creado con atribuciones para conocer con detalle todo el funcionamiento de la administración pública estatal. Sin embargo, sus funciones más de una vez se empalmaron con las de la Secretaría General de Gobierno. Fueron conocidas las pugnas de poder que enfrentó con su titular Manuel González.

En la Secretaría de Educación, los signos de conflictos de intereses fueron denunciados en la gestión de su titular Esthela Gutiérrez, quien fue cuestionada por el "dobleteo de sueldo" - fenómeno que da lugar a conflicto de intereses-, tanto como funcionaria de primer nivel como catedrática de la Universidad Autónoma de Nuevo León (UANL), además de que personal de su primer círculo de confianza contrató a familiares en la misma dependencia. Aunque la extitular adujo que esto no era contrario a la ley en una comparecencia ante representantes del Congreso del Estado (Tapia, 2016). Meses más tarde, en diciembre del 2016, presentó su renuncia como secretaria de Educación, luego de un cuestionado manejo político del conflicto magisterial en el que los maestros de la sección 50 del Sindicato Nacional de Trabajadores de la Educación (SNTE) hicieron varias e intensas jornadas de protesta en el verano del 2016 por la implementación de la reforma educativa.

En el caso del procurador de Justicia del Estado de Nuevo León, Roberto Flores Treviño, estuvo presente desde el inicio de la campaña con Rodríguez Calderón al ser el representante jurídico del candidato independiente ante la Comisión Estatal Electoral de Nuevo León. Aunque desde el inicio fue duramente criticado por los colegios de abogados en el estado, por no tener un perfil reconocido, fue nombrado procurador del Estado. Posteriormente, se descubrió que Flores Treviño había sido procesado en Estados Unidos por girar cheques sin fondos en casinos. A pesar de este antecedente, no fue removido. Esto finalmente ocurrió a principios del 2017 cuando se descubrieron casos de nepotismo en los que su hija, su hermano y su padre trabajaban en diferentes dependencias del Gobierno estatal (Campos, 2017).

Este gobierno en marcha no ha estado exento de la corrupción mostrada y sancionada con evidencia. El primer caso comprobado fue por la compra irregular de 200.000 cobertores que nunca fueron recibidos ni entregados a la población objetivo. Esta transacción tuvo un valor de 30 millones de pesos, en una licitación plagada de irregularidades detectadas por la Contraloría del Estado y como resultado de la cual las sanciones de la Subprocuraduría Anticorrupción contra el exsubsecretario de Administración Rogelio Benavides Pintos y el director de Adquisiciones Gonzalo Padilla Orozco no alcanzan sanciones penales (Tapia, 2017).

Por otra parte, en el ámbito nacional se aprobó la legislación anticorrupción en el año 2016. Por tanto, en el ámbito estatal se aprobó la homologación de la ley, llamándola Ley del Sistema Estatal Anticorrupción 
del Estado de Nuevo León, en julio del 2017. Sin embargo, esta ley no fue por iniciativa del Poder Ejecutivo, sino de un grupo de legisladores de prácticamente todas las bancadas parlamentarias: PRI, PAN, PT, PRD y Movimiento Ciudadano, además de los legisladores llamados independientes (Proyecto Ley del Sistema Estatal Anticorrupción, 2016) viii. Con esto, se crean órganos cuyos titulares serán nombrados por el Congreso de Estado, a saber: Fiscalía General del Estado — desaparece la Procuraduría General del Estado- Fiscalía Especializada en Combate a la Corrupción — sustituye a la Subproduraduría Anticorrupción-y la aparición de la Fiscalía Especializada en Delitos Electorales. Cabe mencionar que la terna enviada por el Poder Ejecutivo fue rechazada en su totalidad por el Congreso y fueron precisamente elegidos los integrantes de la terna consensuada en el interior de los grupos parlamentarios.

\section{Los resultados y la percepción en seguridad}

La inseguridad pública en el Estado ha sido un tema vigente en la agenda pública por lo menos en las dos últimas administraciones estatales. Como ha sido demostrado, los altos índices delictivos son principalmente del fuero común, pero más presencialmente por homicidios relacionados con el crimen organizado que tienen mayor impacto mediático ante la opinión pública (Medellín, 2016b). Por tanto, lo que importa en la discusión es la evidencia empírica que se muestra en los resultados de los tres primeros años de gestión de Rodríguez Calderón: un repunte delictivo y luego una leve mejoría, como se presenta en la figura 1, a partir de los indicadores del Secretariado Ejecutivo del Sistema Nacional de Seguridad Pública.

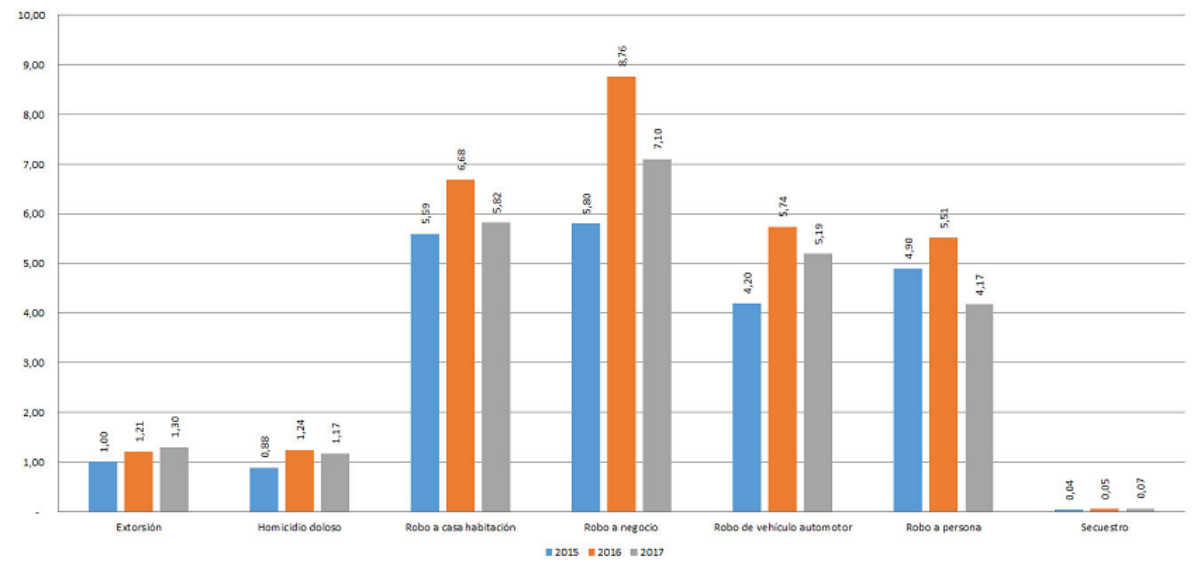

FIGURA 1

Tasa de incidencia delictiva del fuero común por cada 100.000

habitantes en el gobierno de Jaime Rodríguez Calderón (tres años).

Fuente: elaboración propia con información del Secretariado Ejecutivo del Sistema Nacional de Seguridad

Pública (2018), de acuerdo con la nueva metodología para la clasificación de delitos con fines estadísticos

De acuerdo con los datos, los delitos de más alto impacto son el robo a casa-habitación; robo a negocio; robo a vehículo automotor y robo a persona. El 2016, es decir, un año después de que El Bronco asumiera la gubernatura, es el del repunte en todos estos delitos, siendo las tasas más altas en robo a negocio $(8,76 \%)$ y robo a casa-habitación (6,68\%). Posteriormente, aparecen el robo de vehículo $(5,74 \%)$ y el robo a persona $(5,51 \%)$.

No obstante, la percepción sobre inseguridad en el estado, según los datos de la Envipe 2017, aunque no son comparables con los niveles de la administración anterior, siguen siendo altos. En el 2017, el 71\% de los ciudadanos dijo sentirse inseguro en el estado. En el 2016 la cifra fue la más alta de los tres años medibles, un $73,5 \%$ de la población se sintió insegura en el Estado. En esto coincide con la cifra, también de ese año, de los delitos de alto impacto que como hemos señalado fue la más alta en los tres años (tabla 1). 
TABLA 1

Porcentaje de percepción de inseguridad en la entidad federativa de acuerdo con la Encuesta Nacional de Victimización y Percepción sobre Seguridad Pública de México

\begin{tabular}{|l|l|}
\hline Año & Porcentaje (\%) \\
\hline 2013 & 80,2 \\
\hline 2014 & 73 \\
\hline 2015 & 70,7 \\
\hline 2016 & 73,5 \\
\hline 2017 & 71,1 \\
\hline
\end{tabular}

Fuente: elaboración propia con información de Envipe 2017.

Por último, es de hacer notar, en los centros penitenciarios del estado, el llamado "autogobierno de los penales" por parte de los presos, quienes siguen activos como integrantes de las redes delincuenciales y son el poder de facto dentro de los centros de detención — cobro de piso, extorsiones, venta de droga, etc. - Este, sin embargo, no es un problema tan solo del gobierno del estado de Nuevo León, sino que se manifiesta también en otros centros penitenciarios de los estados.

En los tres centros penitenciarios - Cadereyta, Topo Chico y Apodaca, todos con sobrepoblación penitenciaria-, desde septiembre del 2015 hasta el momento en que se escribe este documento, se han presentado ocho episodios con altercados y motines entre los presos. El penal de Topo Chico es el que mayor incidencia presenta, con cinco procesos de alteración, el más trágico en febrero del 2016 cuando murieron 49 internos en un incendio provocado por ellos mismos.

\section{La salida y el regreso a la gubernatura}

Rodríguez Calderón pidió licencia como gobernador de Nuevo León el 20 de diciembre del 2017, dado que su fin primordial era contender como candidato independiente a la presidencia de la República en las elecciones de julio del 2018. Su imagen dentro del estado no era la mejor en el momento, antes bien, después del segundo informe de gobierno un 53\% de los ciudadanos no aprobaban su gestión como gobernador (A. Moreno, 2017).

La solicitud en mención fue presentada al Congreso del Estado, donde el trámite tiene un plazo de seis meses, fue aceptada, y se nombró a Manuel González — secretario general de Gobierno- como gobernador interino. Del total de los cinco aspirantes independientes a la presidencia de la República, siendo los principales Margarita Zavala (expanista), Armando Ríos Píter (experredista) y Jaime Rodríguez Calderón (expriista), solo la primera fue reconocida en primera instancia como candidata independiente por el Instituto Nacional Electoral (INE). En el caso de Rodríguez Calderón, su intento por ser un candidato independiente a la presidencia de la República fue denegado en primera instancia por el INE, el cual invalidó las firmas ciudadanas mínimas que son un requisito indispensable para participar como candidato independiente ${ }^{\mathrm{ix}}$.

En la decisión del INE con respecto al rechazo de las firmas se estimó que

El Bronco entregó poco más de dos millones de firmas ciudadanas, sin embargo 266 mil 357 estaban duplicadas, más de 500 mil tenían inconsistencias; 158 mil 532 estaban simuladas; más de 205 mil eran fotocopias: 158 mil 532 estaban simuladas; más de 205 mil eran fotocopias. Al final quedó con 31 mil 082 firmas debajo de la meta. (Redacción Animal Político, 2018) 
Sin embargo, dentro del recurso de impugnación presentado por Rodríguez Calderón ante el Tribunal Electoral del Poder Juridicial de la Federación, este último resolvió ordenar al INE, en una histórica sentencia "garantista" x , registrar al Bronco como candidato independiente presidencial, al considerar que se habían violado sus derechos de audiencia y garantías de ser votado, pero sin entrar a resolver el fondo de las inconsistencias presentadas (Tribunal Electoral del Poder Judicial de la Federación, 2018). Como sostiene un experto en materia procesal electoral:

La sentencia parte de una falacia fundamental: [se alega que] no se respetó la garantía de audiencia, después de once verificaciones y una general. Doce veces. Doce. No conozco otro procedimiento que tenga igual número de posibilidades de revisión. Por otro lado, si el problema es que no se sabe si 16 mil firmas son válidas o no, los efectos de la sentencia debieron ser: o el tribunal asume en plenitud de jurisdicción el análisis de las 16 mil firmas o le ordena al INE que las verifique. Pero no las da por buena sin saber si lo son [...] La mayoría [de los magistrados] explica su sentencia bajo la premisa del garantismo y el papel del juez constitucional [...] El garantismo implica la maximización de derechos humanos, pero dentro del marco normativo [...] Por otro lado, un juez constitucional no sólo protege derechos humanos, también materializa los principios constitucionales, esos que olvidaron: independencia, imparcialidad, certeza, objetividad, legalidad. (Nieto, 2018)

Es decir, no se resolvió de forma procesal la presunta invalidez de las firmas, sino que se ordenó a la autoridad electoral - de forma inexplicable en términos del debido proceso- subirlo a la boleta. De los resultados electorales en julio del 2018, finalmente Rodríguez Calderón solo logró 2.961 .732 votos, de un total de votación nacional de 56.611 .027 votos, es decir, apenas un 5,23\% (INE, 2018). Al vencer el término de su licencia temporal al cargo, El Bronco regresó a su encargo para terminar su periodo de gobierno.

\section{Conclusión}

El análisis de coyuntura en el caso de Nuevo León se centró en dos vertientes principales, que fueron las condiciones en que un candidato independiente — con pasado partidista - llegó a ser gobernador y cuáles fueron las principales respuestas de gobierno, no en todos los issues de la agenda, sino principalmente en aquellos que representaron una afrenta desde la demanda ciudadana.

En el primer punto, es claro que dada la introducción en la legislación electoral tanto nacional como local de las candidaturas independientes, no se esperó que tuviera un efecto importante en la reconfiguración de los sistemas políticos De ahí que el caso de Nuevo León y su primer gobernador constitucional independientes sea de importancia para el análisis político, a pesar de la debilidades jurídicas en la figura de la candidatura independiente y más aún la ausencia de regulación jurídica de las redes sociales en las campañas electorales. Ahora bien, recalcamos que el uso de las redes sociales fue notoriamente crucial para la victoria del candidato independiente, desbancando todo el anclaje convencional de hacer campañas políticas. De hecho, podemos afirmar con toda propiedad que el estudio de los procesos y campañas político-electorales tiene un antes y un después con la victoria del Bronco en el estado de Nuevo León. Dicha victoria fue tan decisiva en muchos sentidos que siguen las líneas pendientes de investigación para la explicación del realineamiento del electorado y del contraanálisis basado en los grupos de poder empresariales y del crimen organizado que también podrían explicar el enrutado del "poder independiente".

El otro segmento del análisis, como se podrá observar, son los resultados de medir una eficacia gubernamental. Nuestros alcances de investigación aunque fueron solo limitados a dos áreas de la agenda, los resultados señalan que no necesariamente la llegada de un gobernador independiente puede conllevar una eficacia política mayor o por lo menos diferenciada con sus antecesores en temas álgidos, como se mostró en estas páginas. Sin embargo, es necesario aclarar que el análisis es bastante incipiente y no puede ser considerado un marcaje definitivo de la administración estatal, ya que solo se evalúan limitadamente tres años de gobierno. Faltan los últimos tres años de ejercicio, una vez que El Bronco egresó al estado como gobernador. 
Lo que se ha observado como ejercicio de gobierno no puede ser clasificado, de acuerdo con la teoría, como eficaz políticamente para responder a las necesidades de la agenda, sobre todo cuando parte de la élite gobernante que lo acompañó en este primer ciclo no entendió o no quiso responder a esta gran necesidad de cambio que era mostrar resultados decididamente contrarios a la lógica de la corrupción, nepotismo y conflicto de intereses, y exhibir logros altamente positivos en materia de seguridad. En este sentido, si bien hubo una leve mejoría, la percepción de inseguridad de la ciudadanía sigue siendo alta, pues por lo menos siete de cada diez personas consultadas dijeron sentirse inseguras en el estado.

Esto último es quizá es lo más inquietante, en un escenario en el cual el electorado nuevoleonés mayoritariamente confío en que una opción independiente podría acabar con la corrupción y la inseguridad largamente conocidas, por encima de las históricas presencias del PRI o del PAN como gobierno. Y todavía más por el contexto en que la licencia como gobernador del Bronco y su inclusión en la boleta electoral, con una resolución del tribunal electoral que no cumple con los criterios de certeza, objetividad y legalidad, dando razón a la crítica de que su candidatura no tenía nada de independiente y sí mucho de organizada desde las más altas esferas oligárquicas del poder.

En términos gramscianos, puede decirse que el gobierno independiente queda a deber en la construcción de una hegemonía, entendida como "dirección cultural e ideológica", decisiva para asegurar un cambio sustantivo en el quehacer del gobierno. La batalla cultural por dar la confianza a la ciudadanía de que las candidaturas independientes son una opción de gobierno para el cambio quizá se haya quedado lejos de ser una opción razonable para el futuro, por lo menos en el ámbito de esta experiencia local, y qué decir de los resultados a nivel nacional en la experiencia electoral del 2018. Quizá un error estratégico es que esta élite "ciudadana" gobernante no haya entendido que el ejercicio público debe estar enraizado decisivamente en un compromiso democrático para diferenciarse sustancialmente de la élite emanada de los partidos políticos, entendida en su sentido oligárquico. Al final, queda en el imaginario colectivo que son la misma cosa, solo que con sello distinto. Y volvemos de nuevo a Gramsci: entonces este gobierno liderado por Rodríguez Calderón, o no sabe lo que es la hegemonía, o ha fracasado inicialmente en conseguirlo.

\section{Agradecimientos}

Este trabajo se inscribe dentro del Cuerpo Académico Derecho Constitucional y Garantías reconocido por el Programa para el Desarrollo Profesional Docente, para el Tipo Superior de México (Prodep).

\section{Referencias}

Alcántara M. (1995). Gobernabilidad, crisis y cambio. Elementos para el estudio de la gobernabilidad de los sistemas politicos en épocas de crisis y cambio. México: Fondo de Cultura Económica.

Berumen, G. y Medellín L. (2016). Marketing de los candidatos a la gubernatura de Nuevo León en las redes sociales durante el proceso electoral de 2015. Revista Apuntes Electorales, (54), 57-90. Recuperado de http://aelectorale s.ieem.org.mx/index.php/ae/article/view/71

Becerra, R. (1997). Las campañas electorales. Una agenda de acción ciudadana. México: Academia Mexicana de Derechos Humanos.

Becerra, R., Salazar, P. y Woldenberg, J. (1996). La mecánica del cambio politico en México. Elecciones, partidosy reformas. México: Cal y Arena.

Becerril, A. (2017, 17 de abril). "Corrupción envuelve a 11 exgobernadores". Excélsior. Recuperado de http://www.e xcelsior.com.mx/nacional/2017/04/17/1158092.

Campos, G. (2014). Las candidaturas independientes en México. Revista Derecho del Estado, (33), 65-99. Recuperado de http://www.redalyc.org/articulo.oa?id=337632740003. 
Campos, L. (2015a). Jaime Rodríguez Calderón, El Bronco. Claroscuros del hombre que derrotó al sistema. México: Planeta.

Campos, L. (2015b, octubre). “Se les acabó la fiesta a los bandidos", advierte "El Bronco" en su toma de protesta. Proceso. Recuperado de http://www.proceso.com.mx/417200/se-le-acabo-la-fiesta-a-los-bandidos-advierte-el-bronco-e n-su-toma-de-protesta.

Campos, L. (2017). Procurador de Nuevo León renuncia tras escándalos de casinos y nepotismo. Proceso. Recuperado de http://www.proceso.com.mx/475221/procurador-nuevo-leon-renuncia-tras-escandalos-casinos-nepotismo.

Campione, D. (2014). Leer Grasmci. Vida y pensamiento. Buenos Aires: Ediciones Continente.

Cantú, A. (2018). La experiencia de gobiernos divididos en el caso de Nuevo León 2003-2018 (tesis de maestría). Facultad de Derecho y Criminología, Universidad Autónoma de Nuevo León, Nuevo León, México.

Carrizales, D. (2017, 8 de abril). Se desmorona gabinete de "El Bronco". El Universal. Recuperado de http://www.elu niversal.com.mx/articulo/estados/2017/04/8/se-desmorona-el-equipo-del-bronco-se-va-elizondo.

Castillo, L. (2014). Medios y elecciones 2012: viejos y nuevos desafíos para la comunicación política en México. Nóesis. Revista de Ciencias Sociales y Humanidades, 23 (45), 22-48. Recuperado de https://www.redalyc.org/pdf/859/ 85929886004.pdf

Comisión Estatal Electoral. (2015a). Resultados del cómputo estatal 2015. Recuperado de http://ceeresultadosweb.az urewebsites.net/index.html.

Comisión Estatal Electoral. (2015b). Acuerdo del Consejo General de la Comisión Estatal Electoral, relativo a determinar los limites a las aportaciones de carácter privado realizadas a las candidatas y candidatos independientes durante el proceso electoral 2014-2015 derivado del acuerdo INE/CG305/2015. Monterrey, 27 de mayo del 2015. Recuperado de http://www.cee-nl.org.mx/sesiones/2013_2015/acuerdos/20150527.pdf.

Comisión Estatal Electoral. (2015c). Ley Electoral del Estado de Nuevo León. Monterrey: Comisión Estatal Electoral de Nuevo León.

De la Peza, J. (2007). Candidaturas independientes. En D. Nohlen, D. Zovatto, J. Orozco y J. Thompson (comps.), Tratado de derecho electoral comparado de América Latina (pp. 613-625). México: Instituto Interamericano de Derechos Humanos, Universidad de Heidelberg, Idea Internacional, Tribunal Electoral del Poder Judicial de la Federación, Instituto Federal Electoral.

Del Toro, M. (2001). Monopolio de los partidos políticos vs candidaturas independientes: el caso Michoacán SUPJDC-037/2001 desde la óptica del derecho internacional. Revista Justicia Electoral, (17), 119-145. Recuperado de https://tecnologias-educativas.te.gob.mx/RevistaElectoral/content/pdf/a-2001-02-016-120.pdf

Encuesta Nacional de Victimización y Percepción sobre Seguridad Pública (Envipe) (2016). Principales resultados, septiembre 27, 2016. Recuperado de http://www.beta.inegi.org.mx/contenidos/proyectos/enchogares/regulare s/envipe/2017/doc/envipe2017_nl.pdf

Encuesta Nacional de Victimización y Percepción sobre Seguridad Pública (Envipe) (2017). Principales resultados, septiembre 26, 2017. Recuperado de http://www.beta.inegi.org.mx/contenidos/proyectos/enchogares/regulare s/envipe/2017/doc/envipe2017_nl.pdf.

Espinoza, V. (2000). Alternancia y transición política. ¿Cómo gobierna la oposición en México? México: COLEF-Plaza y Valdés Editores.

Estrada, J. (2015, 21 de mayo). Se lava las manos el INE. Reforma. Recuperado de http://www.reforma.com/aplicaci ones/articulo/default.aspx?id $=545160$

González, R. (2015). Candidaturas independientes: entre el individualismo del mexiquense y la endogamia de sus élites políticas. Apuntes Electorales, (52), 83-107. Recuperado de http://aelectorales.ieem.org.mx/index.php/ae/artic le/view/68

Gramsci, A. (1999). Cuadernos desde la cárcel (tomo 3). México: Editorial Era-Benemérita Universidad Autónoma de Puebla.

Hernández, R. (2003). Cambio político y renovación institucional. Las gubernaturas en México. Foro Internacional, (4), 789-821. Recuperado de https://forointernacional.colmex.mx/index.php/fi/article/view/1699/1689 
Hernández, R. (2008). El centro dividido. La nueva autonomía de los gobernadores. México: El Colegio de México.

Instituto Nacional Electoral (INE) (2018). Resultados del Cómputo a nivel distrito y entidad del PEF 2017-2018. Recuperado de https://www.ine.mx/voto-y-elecciones/resultados-electorales/

Lastiri, D. y Carrizales, D. (2017, 27 de enero). Rodrigo Medina, ex gobernador de Nuevo León, entra y sale del penal del Topo Chico en un mismo día. El Universal. Recuperado de http://www.eluniversal.com.mx/articulo/estad os/2017/01/27/rodrigo-medina-entra-y-sale-de-topo-chico-en-un-dia.

Martínez, J. (2015, 17 de mayo). Soy un político incorrecto. El Pais. Recuperado de http://internacional.elpais.com/ internacional/2015/05/15/actualidad/1431725464_658116.html

Mariñez, F. (2009). Compromiso ciudadano. Participación y gestión pública en Nuevo León. México: Fondo Editorial Nuevo León.

Medellín, L. (2011). Liberalización y gobernabilidad en Nuevo León. Monterrey: UANL

Medellín, L. (2016a, 31 de julio). De la elección al “Tornado”. El Norte, p. 8.

Medellín, L. (2016b). La crisis de la seguridad pública y los resultados electorales en el retorno del PRI a Nuevo León. Un análisis retrospectivo. Región y Sociedad, (65), 293-321. Recuperado de http://eprints.uanl.mx/8736/2/36 4-1548-1-PB.pdf

Medellín, L. (2017). Las políticas de seguridad pública en dos sexenios de Nuevo León. Su medición a través de la responsiveness. En F. Betancourt (coord.), Reflexiones sobre el Estado de Derecho, la seguridad pública y el desarrollo de México y América Latina (pp. 157-183). México: UNAM.

Medellín, L. y Murillo, J. (2015). El sistema politico electoral en Nuevo León. Elecciones y efectos en el sistema de partidos politicos (1979-2012). Monterrey: FACDYC-UANL, TEE-CEENL.

Mendoza, D. (2014, 3 de diciembre). Se registra "El Bronco" como candidato a gobernador. Milenio. Recuperado de h ttp://www.milenio.com/politica/se-registra-el-bronco-como-candidato-a-gobernador

Michels, R. (1969). Los partidos políticos: un estudio sociológico de las tendencias oligárquicas de la democracia moderna. Buenos Aires: Amorrortu.

Moreno, A. (2017). Sólo 4 de cada 10 avalan al gobierno del Bronco. El Financiero. Recuperado de http://www.elfin anciero.com.mx/nacional/solo-de-cada-10-avalan-al-gobierno-del-bronco-en-nl.

Moreno, R. (2016). Estrategias electorales en internet: las elecciones presidenciales en México 2012 ¿una referencia para el resto de Latinoamérica? En C. Muñiz y J. Martínez (eds.), Discursos mediáticos en contextos electorales (pp. 205-236). Monterrey: UANL.

Morlino, L. (1985). Cómo cambian los regímenes politicos. Instrumentos de análisis. Madrid: Centro de Estudios Constitucionales.

Nieto, S. (2018). El día que el Tribunal renunció a la imparcialidad. El Universal. Recuperado de http://www.elunive rsal.com.mx/articulo/santiago-nieto-castillo/nacion/el-dia-que-el-tribunal-renuncio-la-imparcialidad

Nuncio, A. (2015). Nuevo León: entre la insularidad y el bipartidismo. El Cotidiano, (193), 23-36.

Pérez, G., Puga, A. y Díaz-Santana, H. (comps). (2009). Memoria histórica de la transición mexicana en México 1977-2007. Documentos básicos para entender el cambio politico (tomos I y II). México: UNAM, Miguel Ángel Porrúa y Senado de la República LX Legislatura.

Proyecto Ley del Sistema Estatal Anticorrupción. (2016). Exposición de Motivos, LXXIV Legislatura, Congreso del Estado de Nuevo León. Recuperado de http://www.hcnl.gob.mx/pdf/proyecto_anticorrupcion.pdf.

Ramos, A. (2016). La constitucionalidad de las candidaturas independientes. México: Tirant lo Blanch.

Ramos, M. (2015, 6 de abril). Reportan falta de equidad en las campañas. El Norte, p. 1a.

Redacción El Universal (2017,30 de marzo). Es mi amigo, dice el "Bronco" de Edgar Veytia, ex fiscal de Nayarit, acusado de narco. El Universal. Recuperado de http://www.eluniversal.com.mx/articulo/estados/2017/03/30/es-mi-a migo-dice-el-bronco-de-edgar-veytia-ex-fiscal-de-nayarit-acusado

Redacción Animal Político (2018). El INE valida las firmas de Margarita Zavala; rechaza los apoyos del Bronco y Ríos Piter. Animal Politico. Recuperado de https://www.animalpolitico.com/2018/03/mexico-bronco-jaime-rodrig uez-ine-firmas/ 
Reveles, F. (2008). Partidos politicos en México. Apuntes teóricos. México: Gernika.

Secretariado Ejecutivo del Sistema Nacional de Seguridad Pública. (2018). Tasas por cada 100 mil habitantes. 1997-2017. Recuperado de http://secretariadoejecutivo.gob.mx/docs/pdfs/tasas\%20por\%20cada\%20100\%20 mil\%20habitantes/Tasas022018.pdf

Soto, L. (2014). Candidaturas independientes. Experiencia zacatecas. Revista Mexicana de Derecho Electoral, (5), 337-363.

Tapia, J. (2016, 2 de marzo). Se defiende secretaria de Educación ante el Congreso de Nuevo León. El Horizonte. Recuperado de http://www.elhorizonte.mx/seccion/se-defiende-secretaria-de-educacion-ante-el-congreso-de$\mathrm{nl} / 1697141$

Tapia, J. (2017, 26 de abril). Arrastra 'firma' de cobertores dudoso historial. El Horizonte, p. 1.

Tejera, H. (2003). "No se olvide de nosotros cuando esté allá arriba". Cultura, ciudadanos y campañas políticas en la ciudad de México. México: UAM-Iztapalapa.

Tribunal Electoral del Poder Judicial de la Federación. (2015). Juicio de Revisión Constitucional Electoral. Expediente: SUP-JRC-656/2015. Octubre 1. Recuperado de http://www.te.gob.mx/Informacion_juridiccional/sesion_pub lica/ejecutoria/sentencias.asp

Tribunal Electoral del Poder Judicial de la Federación. (2018). Instruye TEPJF al INE a emitir nuevo acuerdo sobre registro de Jaime Heliodoro Rodríguez como candidato independiente a la Presidencia de la República. Recuperado de http://portal.te.gob.mx/noticias-opinion-y-eventos/boletin/0/128/2018

Tzili, E. (2016). Masonería en tiempos electorales: el fenómeno del Bronco y la antimasonería en Nuevo León, México (2015). Revista de Estudios Históricos de la Masonería Latinoamericana y Caribeña, (2), 222-246. Recuperado de http://revistas.ucr.ac.cr/index.php/rehmlac/article/view/10351

Woldenberg, J. (2012). Historia minima de la transición democrática en México. México: El Colegio de México.

\section{Notas}

i Fue la sentencia del caso Castañeda Gutman contra México, emitida por la Corte Interamericana de Derechos Humanos, que elevó a "rango nacional" el debate sobre la inclusión de las candidaturas independientes dentro del sistema electoral, aunque ya había otras experiencias estaduales en su lucha por ser reconocidos.

ii Término usado en la jerga periodística para referirse a policías locales que trabajan o tienen relación con el grupo criminal de Los Zetas, dedicados al narcomenudeo, secuestro y extorsión, originarios del vecino estado de Tamaulipas.

iii Sin embargo, de acuerdo con el cotejo legal que pedía la legislación, solo fueron válidas $334 \# 80$ firmas.

iv Aunque en la legislación electoral local existió la posibilidad de tener candidaturas independientes de 1946 a 1976, estas nunca representaron un desafío al régimen priista.

v Hay nuevos estudios sobre este particular proceso electoral, los cuales señalan en análisis de videos de Youtube, que existieron la campaña antimasónica — aludiendo que el Bronco representaba a la masonería - y de miedo político contra Jaime Rodríguez Calderón para inhibir el voto contra él, producido por comunidades religiosas, lo que finalmente tuvo poca recepción (Tzili, 2016).

vi Aclarando que esta inferencia deductiva no toma en cuenta la capacidad del "ciudadano gobernante" en la gestión pública, sino solo la intención de transformarla. Para los retos que enfrenta la participación de la ciudadanía en la gestión pública puede verse el trabajo de Mariñez (2009).

vii Por ejemplo, en otros estados de la República donde hubo elecciones recientes, un lema de campaña exitoso por parte del candidato opositor ha sido "meter a la cárcel al gobernador corrupto". Esto funcionó en Sonora, en la campaña de Claudia Pavlovich (PRI) contra el exgobernador Guillermo Padrés (PAN), actualmente preso, acusado de defraudación fiscal y operaciones con recursos de procedencia ilícita. También en el caso de Chihuahua, el exgobernador César Duarte, acusado de peculado y prófugo de la justicia, fue blanco de la campaña de Javier Corral (PAN), hoy gobernador. En el caso de Veracruz, el exgobernador César Duarte, acusado de peculado y daño al erario, recientemente capturado, fue el tema de campaña del hoy gobernador Miguel Ángel Yunes Linares (PAN). Para una referencia más documentada sobre estos casos, véase el análisis en el que María Amparo Casar - presidenta de Mexicanos contra la Corrupción- menciona en total a once exgobernadores ligados a la delincuencia organizada o acciones de peculado y desvío de recursos (Becerril, 2017). 
viii Está fuera de los alcances de este trabajo analizar la relación del gobierno independiente con el Congreso de Estado. Sin embargo, el caso Nuevo León, por la conformación de mayoría partidista en el Congreso, puede entrar en la tipología de gobierno dividido, esto es, cuando el Poder Ejecutivo no tiene la mayoría legislativa en el Congreso. De los resultados electorales del 2015, aunque se registraron candidatos independientes al Congreso, ninguno ganó. En la LXXIV legislatura hubo diecinueve diputados del PAN; dieciséis del PRI, tres de Movimiento Ciudadano, dos del PVEM, uno del PT y de Nueva Alianza uno. Sin embargo, desde el primer inicio de la legislatura, dos diputados del PAN renunciaron a la filiación parlamentaria y se declararon independientes. No obstante, la relación con el Congreso no ha sido sencilla, ya que al corte de los tres años de gobierno, el Poder Ejecutivo ha vetado al Congreso un total de 38 decretos. Para mayor detalle véase a Cantú (2018).

ix De hecho, fue acusado en más de una ocasión de utilizar la estructura de gobierno para la recolección de firmas ciudadanas.

x El expediente del juicio es el SUP-JDC-186/2018 y acumulado.

* Artículo de reflexión.

\section{Licencia Creative Commons CC BY 4.0}

Cómo citar: Medellín-Mendoza, L. N. (2018. De la victoria de un candidato independiente a los desafíos en la eficacia del desempeño gubernamental: el caso del estado de Nuevo León, México. Papel Político, 23(2). 1-16. https://doi.org/10.11144/Javeriana.papo23-2.vcid 